\title{
Chapter 17 \\ Mitochondrial Activation and Nitric \\ Oxide (NO) Release at Fertilization \\ in Echinoderm Eggs
}

\author{
Tatsuma Mohri and Keiichiro Kyozuka
}

\begin{abstract}
In the cell, mitochondria are important organelles to supply energy for essential cell activities. It is unclear when and how mitochondrial activity increases on fertilization. We refer to mitochondrial activation here as mitochondrial innermembrane hyperpolarization. We measured fluorescence changes in mitochondrial inner-membrane potential $\left(\Delta \Psi_{\mathrm{m}}\right)$ during fertilization in eggs of the echinoderm Hemicentrotus pulcherrimus and the sand dollar Clypeaster japonicus using a mitochondrial dye, MitoTracker Red CMXR (MTR). Increase in fluorescence was detected after insemination in most eggs, which indicates hyperpolarization in $\Delta \Psi_{\mathrm{m}}$. To obtain the relationship between changes in intracellular $\mathrm{Ca}^{2+}\left(\left[\mathrm{Ca}^{2+}\right]_{\mathrm{i}}\right)$ and $\Delta \Psi_{\mathrm{m}}$, simultaneous measurements of $\left[\mathrm{Ca}^{2+}\right]_{\mathrm{i}}$ and $\Delta \Psi_{\mathrm{m}}$ were carried out using MTR and Ca Green dextran. Results showed that an increase in $\Delta \Psi_{\mathrm{m}}$ occurred just after the increase in $\left[\mathrm{Ca}^{2+}\right]_{\mathrm{i}}$. Moreover, microinjection of $1 \mathrm{mM}$ BAPTA suppressed the $\Delta \Psi_{\mathrm{m}}$. These results suggest that the mitochondrial activation is dependent on $\left[\mathrm{Ca}^{2+}\right]_{\mathrm{i}}$ increase. However, nitric oxide $(\mathrm{NO})$ release $(\Delta \mathrm{NO})$ at fertilization in sea urchin eggs is a well-known phenomenon, although its function is not clear. Our previous study demonstrated that $\Delta \mathrm{NO}$ at fertilization occurred at a peak of $\left[\mathrm{Ca}^{2+}\right]_{i}$ and was inhibited by cyanide, a mitochondrial inhibitor, suggesting that $\Delta \mathrm{NO}$ is related to mitochondrial activation. Simultaneous measurements of $\left[\mathrm{Ca}^{2+}\right]_{\mathrm{i}}$ and $\Delta \Psi_{\mathrm{m}}$ revealed that the timing of the increase of $\Delta \Psi_{\mathrm{m}}$ was a little earlier than that of $\Delta \mathrm{NO}$. We conclude both $\Delta \mathrm{NO}$ and changes in $\Delta \Psi_{\mathrm{m}}$ are downstream phenomena caused by sperm-induced $\left[\mathrm{Ca}^{2+}\right]_{i}$ increase. We hypothesize that mitochondrial activation is involved in $\triangle \mathrm{NO}$. Therefore, we are investigating the issue further.
\end{abstract}

\footnotetext{
T. Mohri $(\square)$

National Institutes of Natural Sciences, National Institute

for Physiological Sciences, Okazaki 444-8787, Japan

e-mail: tsmohri@nips.ac.jp

K. Kyozuka

Research Center for Marine Biology, Asamushi, Graduate School of Life Science,

Tohoku University, Asamushi Aomori 039-3501, Japan
} 
Keywords Changes in mitochondrial inner-membrane potential $\left(\Delta \Psi_{\mathrm{m}}\right)$ - Intracellular $\mathrm{Ca}^{2+}\left(\left[\mathrm{Ca}^{2+}\right]_{\mathrm{i}}\right) \cdot \operatorname{Nitric}$ oxide $(\mathrm{NO})$ release $(\Delta \mathrm{NO})$

\subsection{Introduction}

Fertilization is thought to be one of the most remarkable phenomena, in which a large amount of energy is required for a zygote to increase diverse cellular activities leading to structural and qualitative changes for development from a dormant unfertilized state. At fertilization, mitochondria could be significant in increasing various cellular activities, including supplying the energy. Generally, mitochondria have been recognized as multifunctional organelles playing crucial roles in cellular control. Their roles are to provide the main cellular energy in the form of adenosine triphosphate (ATP), to generate and regulate reactive oxygen species, to buffer cytosolic $\mathrm{Ca}^{2+}$, and to regulate apoptosis through a mitochondrial permeability transition pore (Wallace et al. 2010). Therefore, it is assumed that mitochondrial activity should increase accompanying the sequential phenomena, called "egg activation," that comprise changes in intracellular concentration of $\mathrm{Ca}^{2+}\left(\left[\mathrm{Ca}^{2+}\right]_{\mathrm{i}}\right), \mathrm{pH}$, nitric oxide (NO) release $(\Delta N O)$, other second-messenger signals, and cytoplasmic changes, leading to activation of many important enzymes and factors for development. In sea urchins, early studies on respiration demonstrated that a burst of respiration started immediately after fertilization and reached the peak level within 3 min (Foerder et al. 1978; Fujiwara and Yasumasu 1997; Warburg 1908). One third of the respiration at fertilization should be responsible for mitochondrial respiration. However, when and how the mitochondria really activate remains unclear. According to the chemiosmotic theory, measuring mitochondrial inner-membrane potential $\left(\Delta \Psi_{\mathrm{m}}\right)$ has been used as an index of mitochondrial activity because $\Delta \Psi_{\mathrm{m}}$ is utilized to provide energy for ATP production (Solani et al. 2007). Mitochondrial activation is believed to be by changes in hyperpolarization of $\Delta \Psi_{\mathrm{m}}$ (Fujihara et al. 2007). On the other hand, $\Delta \mathrm{NO}$ at fertilization has been reported in sea urchin eggs, although its main function is still obscure (Leckie et al. 2003; Mohri et al. 2008). We found in our previous study that mitochondrial activity has some relationship to $\Delta \mathrm{NO}$ because mitochondrial inhibitors such as $\mathrm{CN}^{-}$and $\mathrm{NaN}_{3}$ inhibited $\Delta \mathrm{NO}$, and an egg stratified by centrifugation showed colocalization by double-staining with the mitochondrial dye MitoRed and the NO indicator DAF-FM DA (Mohri et al. 2008). Therefore, we have investigated the precise relationship between $\Delta \Psi_{\mathrm{m}}$ and $\Delta$ NO.

Here, we report whether mitochondrial activity truly increases after insemination by measuring changes in fluorescence of a mitochondrial inner-membrane potential sensitive dye as $\Delta \Psi_{\mathrm{m}}$ at fertilization using MitoTracker Red CMXR (MTR). For the relationship between $\Delta N O$ and $\Delta \Psi_{\mathrm{m}}$, we show the effect of mitochondrial inhibitors carbonyl cyanide-4-(trifluoromethoxy)phenylhydrazone (FCCP) and $\mathrm{CN}^{-}$on $\Delta \mathrm{NO}$. We also report the relationship between $\left[\mathrm{Ca}^{2+}\right]_{\mathrm{i}}$ and $\Delta \Psi_{\mathrm{m}}$ by simultaneous measurements and show the $\left[\mathrm{Ca}^{2+}\right]_{\mathrm{i}}$ dependency of $\Delta \Psi_{\mathrm{m}}$ by injection of a $\mathrm{Ca}^{2+}$ chelator, 1,2-bis(o-aminophenoxy)ethane- $N, N, N^{\prime}, N^{\prime}$-tetraacetic acid (BAPTA), because $\left[\mathrm{Ca}^{2+}\right]_{\mathrm{i}}$ increase has been reported to induce mitochondrial activation in various 
types of cells such as heart, muscle, liver, kidney, pancreas, and nervous system (Griffiths and Rutter 2009; Gunter and Sheu 2009).

\subsection{Materials and Methods}

\subsubsection{Gametes}

Eggs of the sea urchin Hemicentrotus pulcherrimus and the sand dollar Clypeaster japonicus were used for experiments. Eggs were obtained by injecting 0.2-0.4 ml $0.1 \mathrm{mM}$ acetylcholine into the coelomic cavity. Whole testes removed from males were stored dry at $4{ }^{\circ} \mathrm{C}$. Gametes were used within $10 \mathrm{~h}$ after shedding, only when more than $95 \%$ of the eggs elevated simultaneously with symmetrical fertilization envelopes (FE) and cleaved regularly through the four-cell stage. Experiments were carried out at $20-21{ }^{\circ} \mathrm{C}$ for $H$. pulcherrimus and $24{ }^{\circ} \mathrm{C}$ for $C$. japonicus, respectively, using experimental seawater (ESW) [natural seawater (NSW) passed through a membrane filter $(0.22 \mu \mathrm{m})$, buffered with $10 \mathrm{mM}$ tris(hydroxymethyl)-ethylaminopropane sulfonic acid (TAPS), and adjusted to $\mathrm{pH} 8.2$ with $\mathrm{NaOH}$ ]. The NSW used was collected from surface water off the coast just outside Asamushi Marine Biological Station.

\subsubsection{Measurements of $\Delta \Psi_{m}, \Delta N O$, and $\left[\mathrm{Ca}^{2+}\right]_{i}$}

$\Delta \Psi_{\mathrm{m}}$ was measured as fluorescence changes in eggs loaded with a mitochondrial dye, MitoTracker Red CMXR (MTR; Life Technologies, USA). Eggs were immersed for $10 \mathrm{~min}$ in 1,000 $\mathrm{nM}$ MTR dissolved in ESW. Nitric oxide release $(\Delta \mathrm{NO})$ measurement was carried out in eggs loaded with 12.5-50 $\mu \mathrm{M}$ DAF-FM DA (DAF) for $15 \mathrm{~min}$ or $2.5 \mu \mathrm{M}$ DAR-4M AM (DAR) for $60 \mathrm{~min}$, respectively. For measurement of intracellular $\mathrm{Ca}^{2+}\left(\left[\mathrm{Ca}^{2+}\right]_{i}\right)$, Ca green dextran (CGD) was microinjected into eggs by a slightly modified Hiramoto's method (Hiramoto 1974). Fluorescence images were detected using a high-speed fluorescence imaging system (HSFIS) (AquaCosmos/Ratio; Hamamatsu Photonics, Hamamatsu, Japan) attached to an inverted microscope (TE-300; Nikon, Tokyo, Japan). Some experiments were carried out using a confocal microscope (A1R; Nikon). In the HSFIS, excitation light at $490 \mathrm{~nm}$ and $560 \mathrm{~nm}$ was obtained with a grating monochrometer (POLYCHROME-2; T.I.L.L Photonics, Martinsried, Germany), a dual dichroic beam splitter (FF01-512/630-25; Semrock, Rochester, NY, USA), and a dual emission filter at 512/630 nm were used for a measurement of CGD, DAF, DAR, and MTR fluorescence. Fluorescence images of eggs loaded with those dyes were acquired every 2 and 5 s using a cooled CCD camera (C6790; Hamamatsu Photonics). Processing was performed with Image $\mathrm{J}$ (a public domain image processing software). Changes in fluorescence for both $\Delta \Psi_{\mathrm{m}}$ and $\left[\mathrm{Ca}^{2+}\right]_{\mathrm{i}}$ were expressed on a graph in terms of ratio values, $R\left(F / F_{0}\right)$, where $F$ is a 
background-subtracted fluorescent image of the egg during fertilization and $F_{0}$ a background-subtracted fluorescent image just before insemination. Therefore, the initial value of $R$ is 1 and fractional increase is $R-1$. $\Delta \mathrm{NO}$ is expressed with displacement values as $\mathrm{d} F\left(F-F_{0}\right)$ in a graph because fluorescence of DAF is accumulative and we found it much more suitable to express $\Delta \mathrm{NO}$ as $\mathrm{d} F$ rather than a ratio.

A final concentration of $0.1-2 \mathrm{mM} \mathrm{NaCN}$ and $10-20 \mu \mathrm{M}$ carbonyl cyanide-4-(trifluoromethoxy)phenylhydrazone (FCCP) in ESW was used for experiments. In some experiments, bright-field images were simultaneously monitored with fluorescence measurement using red light at $>640 \mathrm{~nm}$ by another dichroic beam splitter (Chroma Tech, Bellows Falls, VT, USA) and by another CCD camera (C240077; Hamamatsu Photonics) to obtain morphological changes in fertilizing eggs. The images were recorded on a DVD recorder (RDR-HX10; Sony, Tokyo, Japan).

\subsubsection{Experimental Procedure on the Microscopes}

The experimental chamber was made from a $35 \times 10 \mathrm{~mm}$ plastic culture dish lid (Falcon \#1008; Becton Dickinson Japan, Tokyo, Japan) in which a hole of 20-22 mm in diameter was cut out in the bottom and cemented by a circular coverglass (24$26 \mathrm{~mm}$ in diameter) using dental wax. Poly-L-lysine at the concentration of $0.005 \%$ was used to facilitate adhesion of eggs. The chamber was mounted on the stage of the inverted microscope. About five to ten de-jellied eggs loaded with MTR were pipetted into the chamber filled with 3 or $4 \mathrm{ml}$ ESW using a volume-controllable glass pipette connected to a mouthpiece with a silicone tube. For simultaneous measurements of $\left[\mathrm{Ca}^{2+}\right]_{\mathrm{i}}$ and $\Delta \Psi_{\mathrm{m}}$, eggs loaded with MTR were set in the chamber and then microinjected with CGD.

Insemination was carried out by gently pipetting $30 \mu \mathrm{l}$ of diluted suspension of sperm (1-2 $\mu$ l of dry sperm/10 $\mathrm{ml}$ ESW) into the bath $\sim 1 \mathrm{~cm}$ away from the experimental egg after starting simultaneous measurements of fluorescence images and bright-field images. Elevation of the fertilization envelope (FE) and other morphological changes were monitored by bright-field images. After the experiment, egg development was observed at least up to the two-cell stage. Occasionally, development was observed until a higher stage or hatching stage.

\subsection{Results and Discussion}

\subsubsection{Mitochondrial Activation (Inner-Membrane Hyperpolarization) at Fertilization}

Eggs of $H$. pulcherrimus loaded with $50 \mathrm{nM}$ MTR were inseminated and changes in fluorescence reflecting $\Delta \Psi_{\mathrm{m}}$ were measured. At 50-100 s after insemination, an increase in fluorescence was observed (Fig. 17.1A). To examine whether the increase is caused by mitochondrial inner-membrane hyperpolarization, we first 

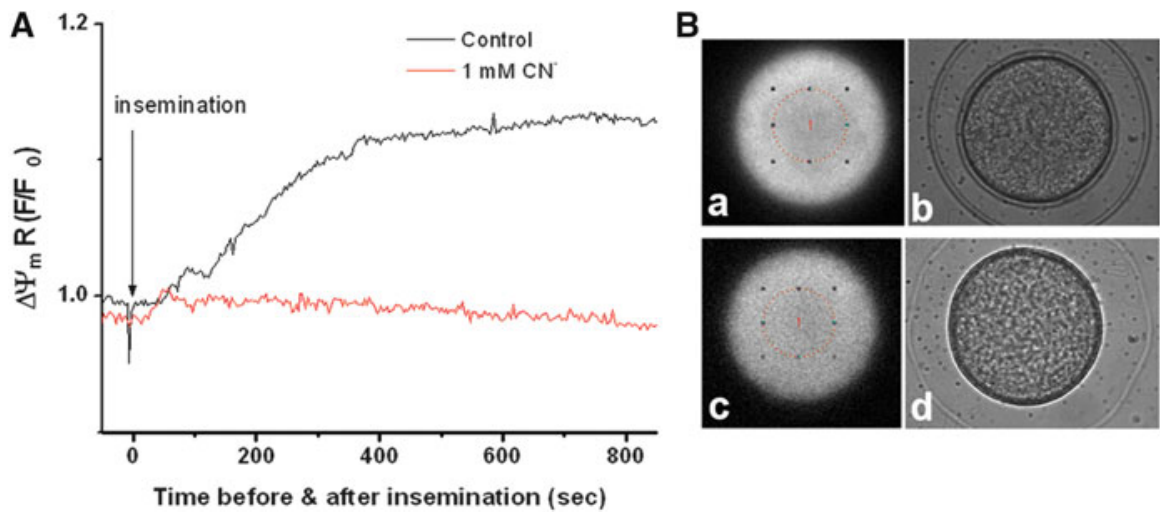

Fig. 17.1 Mitochondrial activation takes place at fertilization. (A) Black and red lines present typical patterns of mitochondrial inner-membrane potential $\left(\Delta \Psi_{\mathrm{m}}\right)$ during fertilization in a control egg and an egg in the presence of $1 \mathrm{mM} \mathrm{NaCN}$, respectively. (B) Corresponding images of fluorescence ( $a$ and $c$ ) and bright field after each experiment ( $b$ and $d$ ). $a$ and $b$ are a control egg, $c$ and $d$ are in the presence of $\mathrm{NaCN}$; they correspond to the black and red lines in a, respectively. Red circle indicates region of interest (ROI) where fluorescence intensities is acquired. Note: Fertilization envelope (FE) elevated in both cases. In $d$, FE looks pale and higher

used MTR in concentrations ranging from 1 to $1,000 \mathrm{nM}$. In most concentrations we tested, the changes in fluorescence were positive (Fig. 17.2). The positive changes indicate hyperpolarization unless the dye, MTR, aggregates within mitochondria and quenches fluorescence. To clarify whether the fluorescence is quenched at the concentration of the dye used, we next applied an uncoupler, FCCP, that dissipates the mitochondrial inner-membrane potential. When, after insemination, 10-20 $\mu \mathrm{M}$ FCCP was applied to eggs showing increase in $\Delta \Psi_{\mathrm{m}}$, the fluorescence signal immediately decreased and then increased but eventually gradually decreased (Fig. 17.3a). When FCCP was added to unfertilized eggs, the fluorescence signal dropped promptly and then continuously decreased. More than 20 min later, those eggs were inseminated. A small increase appeared but eventually showed a gradual decrease in fluorescence (Fig. 17.3b). These results suggest the increase in fluorescence was caused by the hyperpolarization of $\Delta \Psi_{\mathrm{m}}$. All these data suggest that mitochondrial activation takes place at fertilization in sea urchin eggs.

\subsubsection{Inhibition of Mitochondrial Activation $\left(\Delta \Psi_{m}\right)$ by $\mathrm{CN}$ - or FCCP}

$\mathrm{CN}^{-}$is a well-known inhibitor of mitochondrial respiratory complex IV (Fujiwara et al. 2000). Figure 17.4b demonstrates that $\Delta \mathrm{NO}$ was inhibited by $\mathrm{CN}^{-}$as previously reported (Mohri et al. 2008). Because $\mathrm{CN}^{-}$inhibited $\Delta \mathrm{NO}$, we also examined whether $\mathrm{CN}^{-}$affects $\Delta \Psi_{\mathrm{m}}$. We measured $\Delta \Psi_{\mathrm{m}}$ in eggs in the presence of $\mathrm{CN}^{-}$when 


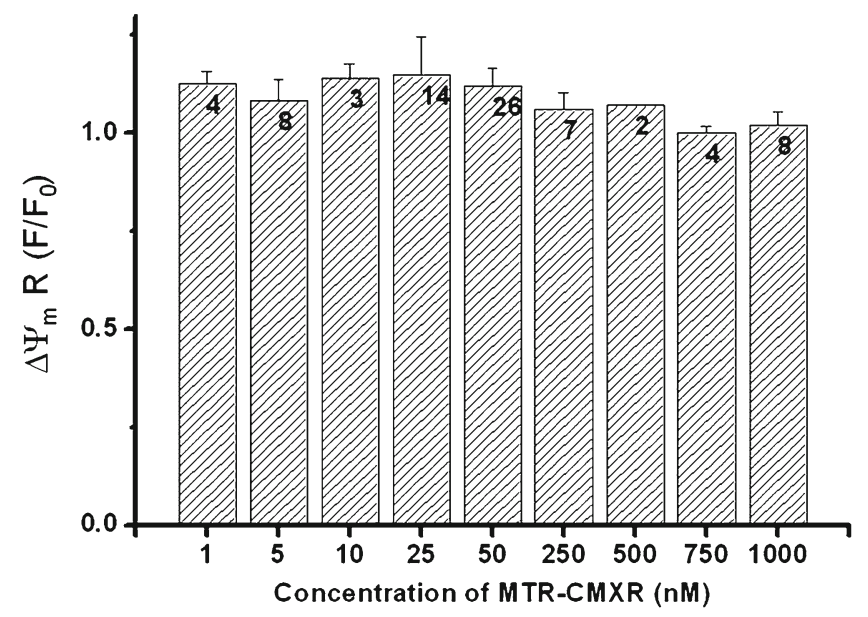

Fig. 17.2 Comparison of $\Delta \Psi_{\mathrm{m}}$ between different concentrations of MitoTracker Red CMXR (MTR). Values of $\Delta \Psi_{\mathrm{m}}$ were measured at $600 \mathrm{~s}$ after insemination and expressed as ratios $($ mean $\pm \mathrm{SD}$ ). The numbers below the $x$-axis represent concentrations of MTR used (in $\mathrm{nM}$ ). Number in each column indicates the number of eggs examined

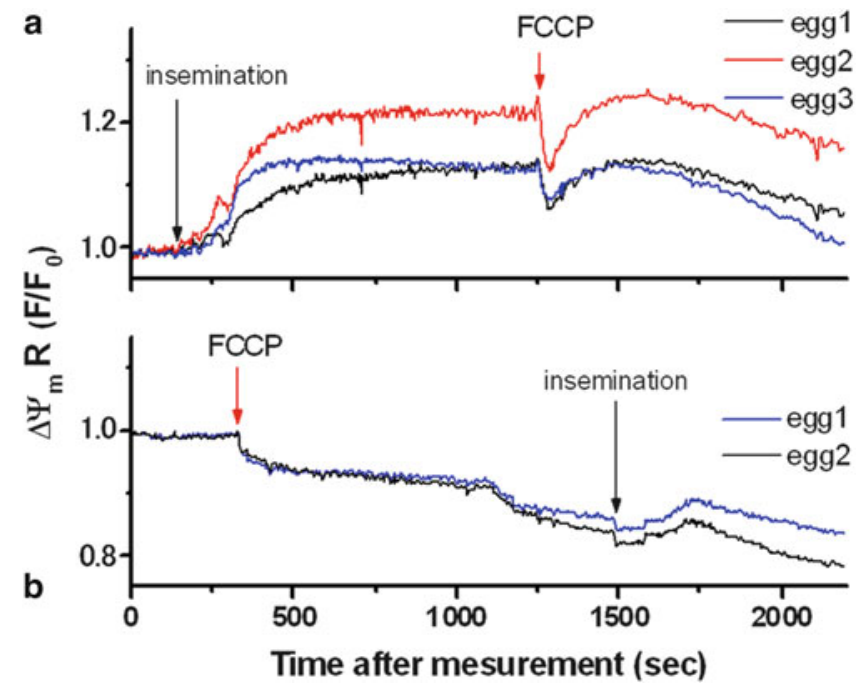

Fig. 17.3 Inhibition of $\Delta \Psi_{\mathrm{m}}$ by carbonyl cyanide-4-(trifluoromethoxy)phenylhydrazone (FCCP). (a) Effect of FCCP addition on $\Delta \Psi_{\mathrm{m}}$ in eggs after fertilization: $20 \mu \mathrm{M}$ FCCP (final concentration) was added in three fertilized Hemicentrotus pulcherrimus eggs in the same chamber. (b) Effect of FCCP addition on $\Delta \Psi_{\mathrm{m}}$ in unfertilized eggs: $20 \mu \mathrm{M}$ FCCP was added in two unfertilized eggs in the same chamber. Insemination was carried out 20 min after addition of FCCP 


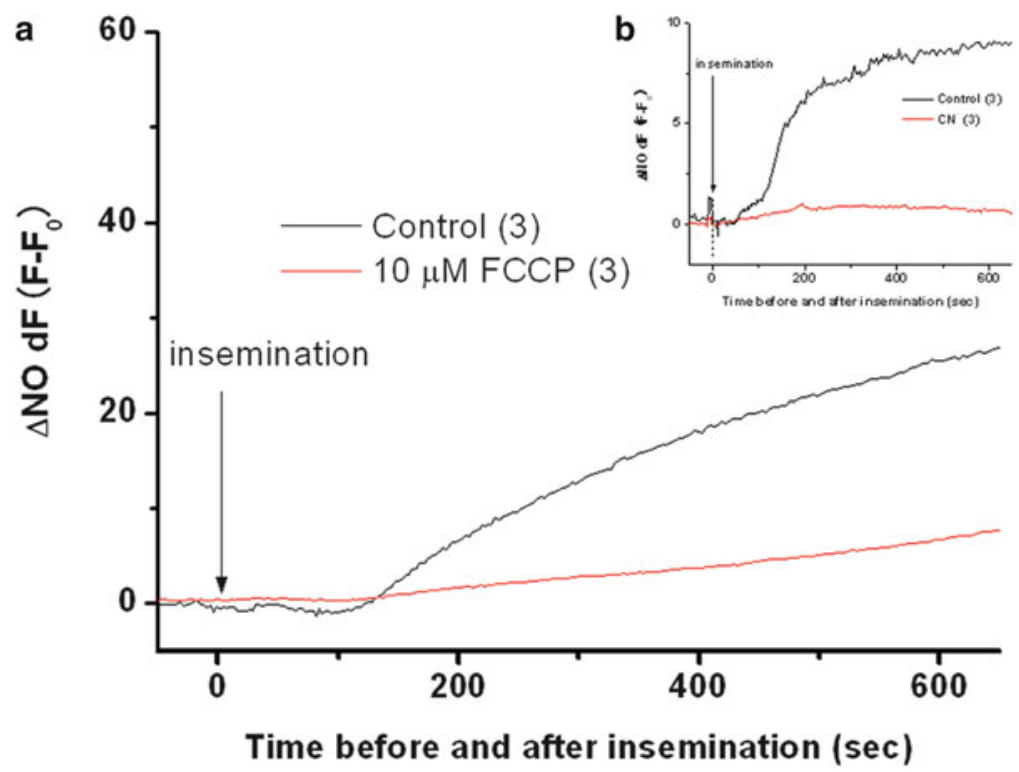

Fig. 17.4 (a) Suppression of $\triangle \mathrm{NO}$ by FCCP. $\Delta \mathrm{NO}$ was measured in the presence of $10 \mu \mathrm{M}$ FCCP. $\Delta$ NOs in control (3) and eggs (3) treated with FCCP were compared. (b) Inhibition of $\Delta$ NO by $\mathrm{CN}^{-} . \Delta \mathrm{NO}$ was measured using DAR. Black and red lines indicate average patterns of $\Delta \mathrm{NO}$ during fertilization in three control eggs and three eggs in the presence of $1 \mathrm{mM} \mathrm{NaCN}$, respectively. Numbers in parentheses indicate the mean number of eggs

inseminated. Eggs loaded with MTR were immersed in 1-2 $\mathrm{mM} \mathrm{NaCN}$ dissolved in ESW for more than $10 \mathrm{~min} . \Delta \Psi_{\mathrm{m}}$ was almost completely inhibited, although very pale, and high FE always appeared (Fig.17.1 A, B-b). Similarly, eggs loaded with MTR were inseminated in the presence of $10 \mu \mathrm{M}$ FCCP after 10-min pretreatment. None of the eggs showed a significant increase in $\Delta \Psi_{\mathrm{m}}$ during fertilization, similar to a late part of the graph after insemination in Fig. 17.3b. Also, $\Delta \mathrm{NO}$ was measured in the presence of $10 \mu \mathrm{M}$ FCCP. Compared to the control, $\Delta \mathrm{NO}$ was significantly suppressed (Fig. 17.4a). Results demonstrated that inhibition of mitochondrial activation by $\mathrm{CN}^{-}$and FCCP induces inhibition of $\Delta \mathrm{NO}$. Taken together, the results suggest $\Delta \mathrm{NO}$ is largely involved in mitochondrial activation.

\subsubsection{Timing of $\Delta \Psi_{m}$ and $\Delta N O$}

In a single measurement of $\Delta \Psi_{\mathrm{m}}$ or $\Delta \mathrm{NO}$, there is uncertainty on the timing of the phenomenon because it is difficult to know the exact time of sperm-egg fusion. Most of the uncertainty is in estimating the time between addition of sperm and cellular fertilization. In addition, there is human error between observation of the egg and recording the clock time. In echinoderm eggs, changes in $\left[\mathrm{Ca}^{2+}\right]_{i}$ always start 


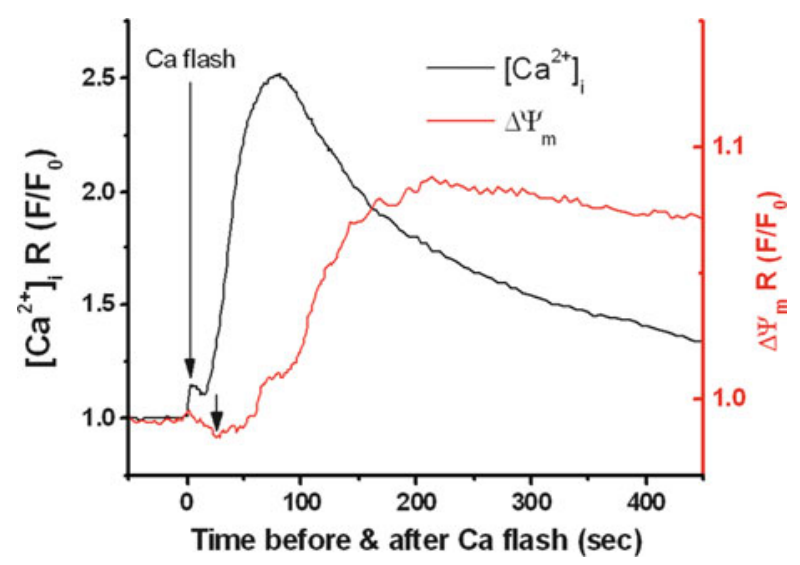

Fig. 17.5 Simultaneous measurements of $\left[\mathrm{Ca}^{2+}\right]_{\mathrm{i}}$ and $\Delta \Psi_{\mathrm{m}} \cdot\left[\mathrm{Ca}^{2+}\right]_{\mathrm{i}}$ and $\Delta \Psi_{\mathrm{m}}$ were simultaneously measured using CGD and MTR. Time 0 was standardized by the occurrence of a "Ca flash," indicating $\mathrm{Ca}^{2+}$ influx from extracellular media through voltage-dependent $\mathrm{Ca}^{2+}$ channels in the egg plasma membrane. A black short arrow indicates a point at which $\Delta \Psi_{\mathrm{m}}$ started to increase just after increasing $\left[\mathrm{Ca}^{2+}\right]_{i}$ at fertilization. A small decreasing part in the beginning of $\Delta \Psi_{\mathrm{m}}$ is possibly an artifact. Details are described in the text

with a small rise in $\left[\mathrm{Ca}^{2+}\right]_{\mathrm{i}}$ called a "Ca flash or cortical flash" (that is induced by $\mathrm{Ca}^{2+}$ influx from the whole surface of the egg through voltage-dependent $\mathrm{Ca}^{2+}$ channels). The Ca flash can be a very good indication of the time point of fertilization. Therefore, to examine more precisely the spatiotemporal relationship between $\left[\mathrm{Ca}^{2+}\right]_{\mathrm{i}}$ and $\Delta \Psi_{\mathrm{m}}$ during fertilization, simultaneous measurements of $\left[\mathrm{Ca}^{2+}\right]_{\mathrm{i}}$ and $\Delta \Psi_{\mathrm{m}}$ were carried out. The patterns of changes in $\left[\mathrm{Ca}^{2+}\right]_{\mathrm{i}}$ and $\Delta \Psi_{\mathrm{m}}$ are shown in Fig. 17.5. Several seconds after the occurrence of the $\mathrm{Ca}$ flash $\left[\mathrm{Ca}^{2+}\right]_{\mathrm{i}}$ began to increase, reached a peak at $80 \mathrm{~s}$, and gradually decreased to one fourth of the peak in $400 \mathrm{~s}$ after the Ca flash. On the other hand, changes in $\Delta \Psi_{\mathrm{m}}$ showed a fraction of decrease several seconds after the Ca flash and then became an significant increase associated with $\left[\mathrm{Ca}^{2+}\right]_{\mathrm{i}}$ rise (Fig. 17.1A). The rise in $\Delta \Psi_{\mathrm{m}}$ started at $25 \mathrm{~s}$ after the Ca flash, during a period of increasing $\left[\mathrm{Ca}^{2+}\right]_{i}$ (small black arrow in Fig. 17.5). Mean value of the increase in $\Delta \Psi_{\mathrm{m}}$ in the simultaneous measurements of $\Delta \Psi_{\mathrm{m}}$ and $\left[\mathrm{Ca}^{2+}\right]_{\mathrm{i}}$ experiments was $29 \pm 8 \mathrm{~s}$ (mean $\pm \mathrm{SD}, n=6$ ). A small fraction of decrease was sometimes observed that may contain an artifact from fluorescence changes by cytoplasmic deformation at fertilization. Similarly, sequentially changing images of $\left[\mathrm{Ca}^{2+}\right]_{\mathrm{i}}$ and $\Delta \Psi_{\mathrm{m}}$ are displayed in Fig. 17.6 where the Ca flash can be seen in the image at 0 in Fig. 17.6a. In separate experiments, $\Delta \Psi_{\mathrm{m}}$ was measured in eggs sandwiched with a coverglass to reduce cytoplasmic deformation using a confocal microscope. $\Delta \Psi_{\mathrm{m}}$ increased at $40 \pm 11 \mathrm{~s}(n=3)$ after insemination without a fraction of decrease (data not shown). The time of increase in $\Delta \Psi_{\mathrm{m}}$ was also close to the value already mentioned using simultaneous measurements of $\Delta \Psi_{\mathrm{m}}$ and $\left[\mathrm{Ca}^{2+}\right]_{\text {i. }}$. 

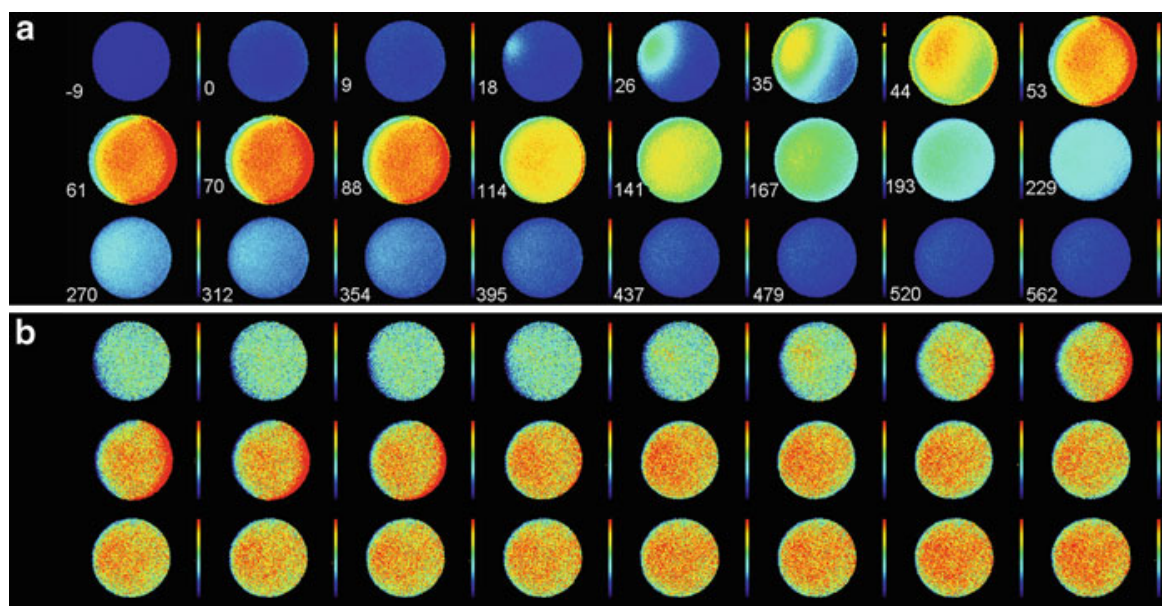

Fig. 17.6 Sequential images of $\left[\mathrm{Ca}^{2+}\right]_{\mathrm{i}}$ (a) and corresponding $\Delta \Psi_{\mathrm{m}}(\mathbf{b})$ before and after Ca flash. Number in the left lower corner of each image indicates seconds (s) before and after Ca flash. The $\left[\mathrm{Ca}^{2+}\right]_{\mathrm{i}}$ images are always $270 \mathrm{~ms}$ ahead of the corresponding $\Delta \Psi_{\mathrm{m}}$ images. Egg of images shown in Fig. 17.6 was different from that in Fig. 17.5

The rise time of $\Delta \mathrm{NO}, 53.5 \mathrm{~s}$, has been previously reported around a peak of $\left[\mathrm{Ca}^{2+}\right]_{\mathrm{i}}$ by the method of simultaneous measurement of $\Delta \mathrm{NO}$ and the activation current $(\mathrm{AC})$ in a voltage-clamped egg because a peak of AC that corresponds to a peak of $\left[\mathrm{Ca}^{2+}\right]_{i}$ (Mohri et al. 1995; Mohri et al. 2008).

Taken together, all these results revealed that $\Delta \Psi_{\mathrm{m}}$ is slightly earlier than $\Delta \mathrm{NO}$, suggesting that $\Delta \mathrm{NO}$ is largely involved in mitochondrial activation.

\subsection{4 $\left[\mathrm{Ca}^{2+}\right]_{i}$ Dependency of $\Delta \Psi_{m}$}

To confirm whether $\Delta \Psi_{m}$ depends on changes in $\left[\mathrm{Ca}^{2+}\right]_{\mathrm{i}}$, an egg microinjected with BAPTA at $1 \mathrm{mM}$ within the egg and the control egg were set in the same field of the chamber and inseminated. The control egg showed normal $\Delta \Psi_{\mathrm{m}}$ whereas the egg injected with $1 \mathrm{mM}$ BAPTA showed no significant increase in $\Delta \Psi_{\mathrm{m}}$ (Fig. 17.7). The egg injected with BAPTA showed no elevation of the fertilization envelope, indicating that sperm-induced changes in $\left[\mathrm{Ca}^{2+}\right]_{i}$ were significantly suppressed. Sperm entries were confirmed by sperm aster formation under bright-field observation after the experiment. Thus, we concluded the increase in $\Delta \Psi_{\mathrm{m}}$ requires, to some extent, an increase in $\left[\mathrm{Ca}^{2+}\right]_{i}$ induced by sperm. It is consistent with earlier research that mitochondrial activation is induced by $\left[\mathrm{Ca}^{2+}\right]_{\mathrm{i}}$ increase in many other cell systems (Griffiths and Rutter 2009). 


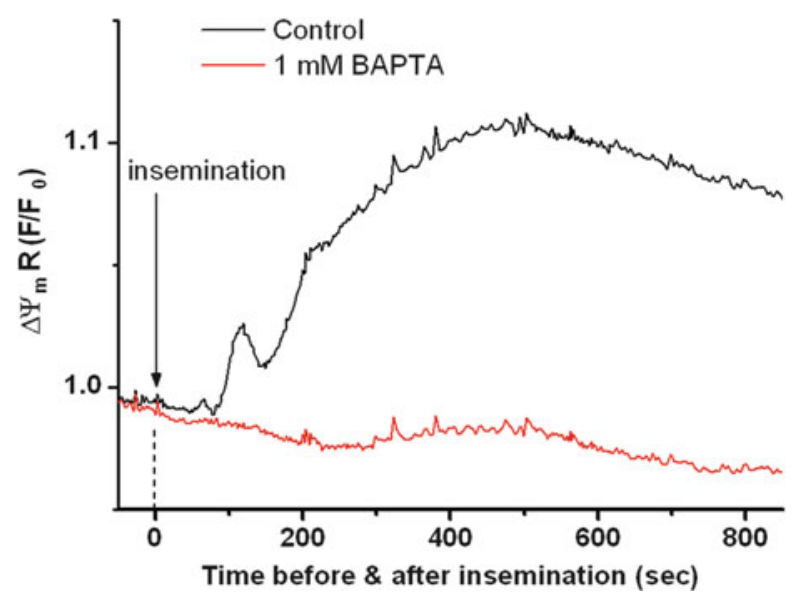

Fig. 17.7 $\Delta \Psi_{\mathrm{m}}$ of control egg and an egg microinjected with $1 \mathrm{mM}$ BAPTA. $\Delta \Psi_{\mathrm{m}} \mathrm{s}$ were measured in a control egg (black trace) and an egg (red trace) injected with $1 \mathrm{mM} \mathrm{BAPTA}$ (final concentration in the egg) in the same chamber set in the stage of the microscope. Declining trace in control during 120-160 s was an artifact caused by fluorescence change by deformation of the egg while FE was forming

\subsection{Conclusion}

In the present study, we found that mitochondrial activation indicating hyperpolarization of $\Delta \Psi_{\mathrm{m}}$ occurred downstream to the increase of $\left[\mathrm{Ca}^{2+}\right]_{\mathrm{i}}$ during fertilization, and that it started after the $\left[\mathrm{Ca}^{2+}\right]_{i}$ rise in sea urchin and sand dollar eggs. We confirmed $\left[\mathrm{Ca}^{2+}\right]_{\mathrm{i}}$ dependency of $\Delta \Psi_{\mathrm{m}}$ by an experiment in which an injection of BAPTA inhibited $\Delta \Psi_{\mathrm{m}}$. Therefore, $\Delta \Psi_{\mathrm{m}}$ was apparently $\left[\mathrm{Ca}^{2+}\right]_{\mathrm{i}}$ dependent, similar to $\Delta \mathrm{NO}$, as previously reported (Leckie et al. 2003; Mohri et al. 2008). Mitochondrial inhibitors $\mathrm{CN}^{-}$and FCCP inhibited both $\Delta \mathrm{NO}$ and $\Delta \Psi_{\mathrm{m}}$, suggesting that mitochondria participate in $\Delta \mathrm{NO}$ and may well regulate $\Delta \mathrm{NO}$. Furthermore, simultaneous measurements of $\left[\mathrm{Ca}^{2+}\right]_{\mathrm{i}}$ and $\Delta \Psi_{\mathrm{m}}$ revealed mitochondrial activation took place slightly earlier than $\Delta \mathrm{NO}$, suggesting that mitochondria may be responsible for $\Delta \mathrm{NO} . \Delta \mathrm{NO}$ has generally been considered to be produced by cytosolic NO synthase (NOS) (Thaler and Epel 2003). Our data suggest that mitochondria may have their own separate generation system of NO such as mitochondrial NOS, mtNOS (Finocchietto et al. 2009). The present data suggest mitochondrial activity unmistakably participates in $\Delta \mathrm{NO}$. Therefore, we are now continuing this investigation.

Open Access: This article is distributed under the terms of the Creative Commons Attribution Noncommercial License which permits any noncommercial use, distribution, and reproduction in any medium, provided the original author(s) and source are credited. 


\section{References}

Finocchietto PV, Franco MC, Holod S, Gonzalez AS, Converso DP, Antico Arciuch VG, Serra MP, Poderoso JJ, Carreras MC (2009) Mitochondrial nitric oxide synthase: a masterpiece of metabolic adaptation, cell growth, transformation, and death. Exp Biol Med (Maywood) 234(9):1020-1028

Foerder CA, Klebanoff SJ, Shapiro BM (1978) Hydrogen peroxide production, chemiluminescence, and the respiratory burst of fertilization: interrelated events in early sea urchin development. Proc Natl Acad Sci USA 75(7):3183-3187

Fujihara T, Nakagawa-Izumi A, Ozawa T, Numata O (2007) High-molecular-weight polyphenols from oolong tea and black tea: purification, some properties, and role in increasing mitochondrial membrane potential. Biosci Biotechnol Biochem 71(3):711-719

Fujiwara A, Kamata Y, Asami K, Yasumasu I (2000) Relationship between ATP level and respiratory rate in sea urchin embryos. Dev Growth Differ 42(2):155-165

Fujiwara A, Yasumasu I (1997) Does the respiratory rate in sea urchin embryos increase during early development without proliferation of mitochondria? Dev Growth Differ 39(2):179-189

Griffiths EJ, Rutter GA (2009) Mitochondrial calcium as a key regulator of mitochondrial ATP production in mammalian cells. Biochim Biophys Acta 1787(11):1324-1333

Gunter TE, Sheu SS (2009) Characteristics and possible functions of mitochondrial $\left.\mathrm{Ca}^{(2+}\right)$ transport mechanisms. Biochim Biophys Acta 1787(11):1291-1308

Hiramoto Y (1974) A method of microinjection. Exp Cell Res 87(2):403-406

Leckie C, Empson R, Becchetti A, Thomas J, Galione A, Whitaker M (2003) The NO pathway acts late during the fertilization response in sea urchin eggs. J Biol Chem 278(14):12247-12254

Mohri T, Ivonnet PI, Chambers EL (1995) Effect on sperm-induced activation current and increase of cytosolic $\mathrm{Ca}^{2+}$ by agents that modify the mobilization of $\left[\mathrm{Ca}^{2+}\right]_{i}$. I. Heparin and pentosan polysulfate. Dev Biol 172(1):139-157

Mohri T, Sokabe M, Kyozuka K (2008) Nitric oxide (NO) increase at fertilization in sea urchin eggs upregulates fertilization envelope hardening. Dev Biol 322(2):251-262

Solani G, Sgarbi G, Lenaz G, Baracca A (2007) Evaluating mitochondrial membrane potential in cells. Biosci Rep 27:11-21

Thaler CD, Epel D (2003) Nitric oxide in oocyte maturation, ovulation, fertilization, cleavage and implantation: a little dab'll do ya. Curr Pharm Des 9(5):399-409

Wallace DC, Fan W, Procaccio V (2010) Mitochondrial energetics and therapeutics. Annu Rev Pathol 5:297-348

Warburg O (1908) Beobachtungen uber die oxydationsprozesse im seeigelei. Hoppe-Seyler's Z Physiol Chem 66:1-16 